\title{
Editorial
}

\section{What is the true role of surfactant in the lung?}

The surfactant identified ${ }^{1}$ in the lung has been accorded two major roles. Firstly, it is believed that surfactant "reduces the work of expanding the lung with each breath" by reducing surface tension at the air-alveolar interface and secondly, in so doing, it also tends to "keep alveoli dry" by reducing the tendency to "suck fluid into the alveolar spaces from the capillaries." Since these roles are now widely accepted to the point where they are quoted in standard medical textbooks, including the most recent in Respiratory Physiology, ${ }^{2}$ it would seem appropriate to start by assessing their validity in the light of recent experimental evidence.

The "mechanical" advantages attributed to surfactant have been based upon the assumption that anything which can reduce the pressure needed to inflate the excised lung is going to make breathing easier. ${ }^{3}$ Since von Neergaard ${ }^{4}$ first showed that the pressure differential needed to inflate an excised lung with air is reduced about tenfold when using saline for inflation-that is, when the interface between the air and aqueous hypophase is eliminated-there has been no doubt that the. interface itself provides the predominant contribution to lung recoil. Hence it is argued that any factor which can reduce surface forces should make breathing easier and this would include surfactants, especially if they reduce the interfacial tension at the alveolar surface from 50 dynes $/ \mathrm{cm}$ for plasma to the very low value of 2 dynes $/ \mathrm{cm}$ recorded in vitro for synthetic dipalmitoyl lecithin (DPL) and even for lung extracts. ${ }^{5}$ Dipalmitoyl lecithin has been identified as one of the most surface active substances present. ${ }^{1}$

While this may offer a plausible explanation for the presence of DPL and other surfactants, it must be remembered that, in normal breathing, the lung is not excised but is located within the thoracic cavity. Hence it is mechanically coupled to the chest wall and diaphragm-also elastic structures-with no opportunity for independent

Address for reprint requests: Professor BA Hills, Department of Anesthesiology, The University of Texas Health Science Center at Houston, 6431 Fannin, Room 5020, PO Box 20708, Houston, Texas 77025. movement. The lung tends to "pull in" the chest wall with the same force that the chest wall exerts to inflate the lung such that, at rest, they reach a position of mutual mechanical equilibrium and then exchange potential energy during the respiratory cycle. It does not necessarily make breathing any easier if lung recoil is reduced by greatly reducing surface tension in locating a potent surfactant at the alveolar-air interface. In other words, a position of mechanical equilibrium reached at lower lung recoil (larger FRC) need not necessarily offer a more efficient pressurevolume cycle during active ventilation and, hence, less work of breathing.

Before discussing the vital question of work of breathing, the above criticisms of the popular belief that surfactant is present primarily to reduce surface tension is somewhat academic, since the lung and chest wall adopt a particular point of mechanical equilibrium at normal compression of the alveolar surface-that is, at FRC-whatever the theoretical reasons. Hence another approach to this issue is to ask what surface tension provides the lung recoil needed to realise the normal state.

Since the original work of von Neergaard, ${ }^{4}$ the Laplace equation $(\Delta \mathrm{P}=2 \gamma / \mathrm{r})$ has been used almost universally $^{5}$ to relate surface tension $(\gamma)$ and radius $(r)$ to the pressure differential $(\Delta P)$ needed to overcome surface forces as though the alveolus were a bubble. At FRC, $\Delta \mathrm{P}$ is about $7 \mathrm{~cm}$ wg for an alveolar diameter (2r) ranging from 75 to $300 \mu \mathrm{m}$ for a geometric mean of $150 \mu \mathrm{m}$. For these figures the Laplace equation gives a surface tension $(\gamma)$ of 26 dyne/cm for the lowest lung volume during normal breathing. This is many fold higher than the minimal values of about 2 dyne $/ \mathrm{cm}$ quoted from conventional studies ${ }^{5}$ of surfactant. However, that value is encompassed by the range of 24-34 dyne/cm for the surface tension of DPL films actually measured in vitro under "physiological" conditions. ${ }^{6}$ These include simultaneous humidification, and selection of body temperature, respiratory frequency, and amplitude of area change along with physiological DPL concentrations and $\mathrm{pH}$ of plasma or Ringer's 
solution as the aqueous hypophase. This indicates that, by reducing surface tension from 50 dyne $/ \mathrm{cm}$ for plasma to 24-34 dyne $/ \mathrm{cm}$, surfactant is playing a much more modest role than is generally believed in this regard and yet the change from 34 to 24 dyne/cm found for a "physiological" area change is still twice the minimum needed to impart alveolar stability, another role attributed to surfactant ${ }^{5}$ and not disputed here. It is most interesting that higher values of surface tension have also been found using "wetted" surfactant. ${ }^{3}$

Before leaving mechanical aspects to discuss what other roles surfactant may play, the vital issue concerns the source of the work of breathing. Under no-flow conditions, this has been largely attributed to the lung itself, since the excised lung displays wide pressure-volume loops otherwise known as compliance hysteresis. This has, in turn, been widely attributed to the surfactant, ${ }^{7}$ since surfaces with films of those surfactants prepared in vitro show wide loops in surface tension versus surface area $(\gamma-\mathrm{A})$ when cycled under the same conventional conditions ${ }^{5} 7$ as were used in reporting the low values of the order of 2 dyne/cm mentioned earlier. Thus there has been something of a contradiction in the literature in that surfactant has been claimed, on the one hand, to make breathing easier by reducing surface tension and yet, on the other hand, blamed for much of the work of breathing by virtue of its contribution to compliance hysteresis. If the earlier argument advanced in this paper is essentially correct, then we should not consider absolute values of surface tension at the airalveolar interface, but the hysteresis represented by the $\gamma$-A loops. However the very wide $\gamma-\mathrm{A}$ loops -that is, gross surface hysteresis-usually attributed to surfactant ${ }^{57}$ raises the basic question as to why the body would incorporate cells in the lung for producing a surfactant which would increase the area of the pressure-volume loop and so cause more work of breathing, the all-important parameter in both health and disease. It was, therefore, most reassuring to find that, when the surface tension of DPL films was measured under "physiological" conditions, there was virtually no $\gamma-\mathrm{A}$ hysteresis. ${ }^{6}$ This restores confidence in the general rule that the body would not produce a compound to make it work harder.

Compliance hysteresis of the excised lung-the phenomenon leading to studies in surface hysteresis-can be attributed to "geometric irreversibility" of lung surfaces ${ }^{89}$ rather than to surfactant per se. This is fairly obvious from observing the contortions of excised lung segments during in- flation or simply comparing the much wider pressure-volume loops from excised lung with those for interpleural pressure with the lungs in situ-as reflected by oesophageal pressure. Moreover, our latest experimental evidence tends to confirm this concept that compliance hysteresis of the excised lung is largely an artefact introduced when that lung is excised from the body and allowed to change volume unrestricted by the geometric constraints imposed by the thoracic cavity.

The above arguments de-emphasise the direct mechanical role of surfactant-other than as a lubricant and alveolar stabilising agent-and therefore lead to one vital question. If DPL and other agents cause only modest reductions in surface tension, why are they present at all, and for what other reason would the body provide a substance which is otherwise so highly surface active? One clue may be provided by the finding that DPL can induce a contact angle at epithelial surfaces. ${ }^{10}$ In surface physics, ${ }^{11}$ a contact angle signifies non-wettability as observed when water placed upon an oily surface forms droplets rather than a continuous film. The above finding is not surprising when considering that, although technically amphoteric, DPL is effectively a cationic surfactant-unlike anionic detergents or nonionic Tween 20 used in previous lung studies. ${ }^{5}$

From a chemical standpoint, the DPL molecule has the two major features which characterise cationic surfactants-a particular group of surface active agents which have been under intense investigation over the last decade or so as their very important industrial applications ${ }^{12}$ have been realised. The first structural feature is the quaternary nitrogen ion whose positive charge enables the whole molecule to be attached quite firmly to any surface containing negative charges such as abound in the pulmonary membrane. The second feature is the long hydrocarbon chains which are then orientated outwards to provide a hydrophobic surface with consequent lack of wettability. In industry, one of the major applications of such compounds concerns water repellency where the textile chemists can treat fabrics containing negative surface charges (grafted cotton) to render it waterproof and yet, as they say, ${ }^{13}$ "able to breathe" - that is gases and water vapour can diffuse freely in both directions and yet liquid water cannot enter the fabric. ${ }^{13}$ The best cationic surfactants for this purpose induce a large contact angle with the least reduction in surface tension, ${ }^{11}$ exactly the combination of properties induced by DPL under physiological con- 
ditions. ${ }^{6}$ Hence it is tempting to speculate that one of the roles of DPL in the lung is as a waterrepellent in helping to keep the alveolar surfaces dry. DPL has often been implicated in oedema. $^{2}$ The above mechanism would seem rather more plausible than any based upon capillarity since any feature of the surface in reducing the tendency for fluid to enter the vascular side of a "pore" would equally reduce the tendency for the fluid to emerge at the other end.

Another reason for considering lung surfactants as waterproofing agents is that other cationic surfactants applied to textiles with negative surface charges can raise the "water entry pressure" to over one atmosphere ${ }^{13}$ - that is, to several orders of magnitude in excess of pulmonary arterial pressure. Thus DPL in the lung would need to have only one per cent of the efficiency of other cationic surfactants in those systems for it to keep the airways dry. In vivo evidence to support this new concept is provided by the finding that agents selected as antifoams, for example, some silicones, offer appreciable protection against acute pulmonary oedema. ${ }^{14}$ This is most pertinent since antifoams act by inducing a contact angle and, hence, most of those substances induce water-repellency when applied to a surface. This possible role for lung surfactant may have come at an opportune time now that the validity of the Starling hypothesis, and the whole question of pulmonary homeostasis, is under intense reexamination. ${ }^{15}$ Since the body seldom relies upon a single mode of preventing any untoward effect, waterproofing could well provide a back-up defence against alveolar oedema.

Viewed alone, however, the above hypothesis might appear to be deficient in explaining how homeostasis would be re-established after a "break-through" such as occurs after an episode of intense pulmonary hypertension. ${ }^{16}$ This can be compared to the boy who touches the inside of a tent during a rainstorm and starts a drip which cannot be stopped. It is interesting to note that re-establishment of homeostasis takes much longer than predicted on the basis of oncotic pressures. In attempting to explain the repair of a "break-through" by the waterproofing model, it must be remembered that, unlike the example of the tent in the rain, alveolar type 2 cells are continuously secreting surfactant on to the alveolar surface while similar cationic surfactants are often used industrially as "de-watering" agents. ${ }^{12}$ In this regard, they have wide application as the additives which make oils "oily" and represent the ingredient of lubricants and underseals for cars which enable those compounds to make true contact with the surface which they lubricate or protect from corrosion. Essentially they act by displacing the thin film of water which coats most surfaces by exploiting the strong affinity of the charged quaternary nitrogen for the surface, thus leaving the hydrophobic tails facing outwards to provide a new surface highly compatible with the oil. In so doing, they displace the water film just as one could envisage DPL mobilising any transmitted water wetting the alveolar lining for its subsequent removal via the mucociliary escalator system.

The implied tendency for surfactant to get beneath the aqueous hypophase and "mobilise" it in preference to spreading over its interface with air is consistent with electron microscopic studies. Most of these do not show macromolecules at this interface nor a continuous liquid lining to the alveoli but "pooling" of the fluid," 17 not unlike the distribution of water placed upon a rough hydrophobic surface. Thus water repellency imparted by surfactant would not only aid homeostasis but, by breaking up the surface layer, would also improve gas transfer by reducing the bloodair barrier just as water repellents can be used in steam engines to induce "dropwise" condensation $^{18}$ and so improve heat transfer at the condenser walls.

Yet another and most important commercial application of cationic surfactants is their use as "fabric softeners". ${ }^{12}$ Their absorption on to the surfaces of fibres causes the filaments of the yarn to separate, thus imparting a much softer "feel" to the garment, a quality much exploited in commercial advertising. Just as it is highly desirable to prevent filaments from clumping together in a yarn, so it would be most desirable to maintain the integrity of the extremely delicate architecture of the lung with microvilii freely protruding into alveolar air to provide the enormous surface area needed for efficient gas transfer. It is therefore tempting to speculate upon how a deficit of surfactant could lead to a gradual degradation of the fine structure of the lung such as observed in emphysema. Indeed, from looking at back-scatter electromicrographs of lungs ${ }^{17}$ and textile fibres, there is a marked similarity between pulmonary capillaries evenly suspended in air and the fibres of a yarn treated with a cationic surfactant.

Yet another interesting industrial application of cationic surfactants is their wide use as surface fungicides and bactericides. ${ }^{12}$ This immediately implies that such roles are possible for DPL in the lung-although unlikely. 
The foregoing discussion emphasises the point that the lung surfactant system is essentially cationic and may have many additional roles in which the conventional emphasis upon reduction of surface tension at the air-alveolar interface may be something of a "red herring". While these are all beneficial properties under normal conditions, there could be adverse effects under unnatural circumstances, such as after surgery or during decompression of a diver, when the pulmonary circulation would filter out any air emboli entering the venous system. Lung surfactant gradually migrates to the surface of these bubbles ${ }^{19}$ when reduction of their surface area upon dissolving or recompression could facilitate their release as arterial emboli. If the surface tension induced by such surfactants were not much higher than quoted conventionally, ${ }^{5}$ - that is, not up to "physiological" values" -arterial air embolism might be a much more common clinical finding. ${ }^{16}$

BRIAN A HILLS

Department of Community and Occupational Medicine

The Medical School

University of Dundee

Dundee

\section{References}

1 Pattle RE. Surface lining of lung alveoli. Physiol Rev 1965; 45:48-79.

2 West JB. Respiratory physiology. The essentials. Second edition. Baltimore: Williams and Wilkins, 1979.

3 Bangham A. Breathing made easy. New Scientist 1980; 85:408-10.

4 Neergaard $K$ von. Neue Auffassungen über einen Grundbegriff der atemmechanik. Die retraktionskraft der Lunge, abhangig von der Oberflachenspannung in der Alveolen. $Z$ ges Exp Med 1929; 66:373-94.

5 Clements JA, Tierney DF. Alveolar instability associated with altered surface tension. In Fenn WO, Rahn A (eds). Handbook of physiology:
Respiration vol II. Washington: Am Physiol Soc, 1966:1565-83.

6 Barrow RE, Hills BA. Surface tension induced by dipalmitoyl lecithin in vitro under physiological conditions. J Physiol 1979; 297:217-27.

7 Clements JA. Surface tension of lung extracts. Proc Soc Exp Biol Med 1957; 95:170-2.

8 Hills BA. Geometric irreversibility and compliance hysteresis in the lung. Respir Physiol 1971; 13:50-61.

9 Daly BDT, Parks GE, Edmonds $\mathrm{CH}$, Hibbs CW, Norman JC. Dynamic alveolar mechanics as studied by videomicroscopy. Respir Physiol 1975; 24:217-32.

10 Hills BA, Barrow RE. The contact angle induced by DPL at pulmonary epithelial surfaces. Respir Physiol 1979; 38:173-83.

11 Adamson AW. The physical chemistry of surfaces. Second edition. New York: Interscience, 1967:476-8.

12 ABM. Quaternary ammonium compounds. Technical Bulletin O2/HSC. Stockport: ABM Chemicals, 1980.

13 Tanner JC. Comfort, breathability, and GoreTex laminates. Company report of Gore-Tex, Elkton, Maryland, 1979.

14 Luisada AA, Cardi L. Further studies with antifoaming agents in experimental pulmonary edema. Circ Res 1955; 3:510-3.

15 Staub NC. Pulmonary edema. Physiol Rev 1974; 54:678-811.

16 Effors RM, Chang RSY. Distribution of water and proteins in the lung in pulmonary edema. In: Fishman AP, Renkin EM (eds). Pulmonary edema. Washington: American Physiological Society, 1979.

17 Weibel ER, Bachofen H. Structural design of the alveolar septum and fluid exchange. In: Fishman AP, Renkin EM (eds). Washington: American Physiological Society, 1979.

18 Drew TB, Nagle WM, Smith WQ. The conditions for drop-wise condensation of steam. Trans Am Inst Chem Eng 1935; 31:605-21.

19 Hills BA, Butler BD. Migration of surfactant to pulmonary air emboli. Proceedings of 7th Symposium on Underwater Physiology. Washington: FASEB, 1980. 